Draft Version January 20, 2016

Preprint typeset using $\mathrm{IAT}_{\mathrm{E} X} \mathrm{X}$ style emulateapj v. 5/2/11

\title{
THE RADIAL AND ROTATIONAL VELOCITIES OF PSO J318.5338-22.8603, A NEWLY CONFIRMED PLANETARY-MASS MEMBER OF THE $\beta$ PICTORIS MOVING GROUP
}

\author{
K. N. Allers \\ Department of Physics and Astronomy, Bucknell University, Lewisburg, PA 17837, USA; k.allers@bucknell.edu \\ J. F. Gallimore \\ Department of Physics and Astronomy, Bucknell University, Lewisburg, PA 17837, USA \\ Michael C. LiU \\ Institute for Astronomy, University of Hawaii, 2680 Woodlawn Drive, Honolulu, HI 96822, USA \\ TREnT J. DupuY \\ The University of Texas at Austin, Department of Astronomy, 2515 Speedway C1400, Austin, TX 78712, USA \\ Draft version January 20, 2016
}

\begin{abstract}
PSO J318.5338-22.8603 is an extremely-red planetary-mass object that has been identified as a candidate member of the $\beta$ Pictoris moving group based on its spatial position and tangential velocity. We present a high resolution $K$-band spectrum of PSO J318.5338-22.8603. Using a forward-modeling Markov Chain Monte Carlo approach, we report the first measurement of the radial velocity and $v \sin (i)$ of PSO J318.5-22, $-6.0_{-1.1}^{+0.8} \mathrm{~km} \mathrm{~s}^{-1}$ and $17.5_{-2.8}^{+2.3} \mathrm{~km} \mathrm{~s}^{-1}$, respectively. We calculate the space velocity and position of PSO J318.5-22 and confirm that it is a member of the $\beta$ Pictoris moving group. Adopting an age of $23 \pm 3 \mathrm{Myr}$ for PSO J318.5-22, we determine a mass of $8.3 \pm 0.5 M_{\mathrm{Jup}}$ and effective temperature of $1127_{-26}^{+24} \mathrm{~K}$ using evolutionary models. PSO J318.5338-22.8603 is intermediate in mass and temperature to the directly-imaged planets $\beta$ Pictoris $\mathrm{b}$ and 51 Eridani b, making it an important benchmark object in the sequence of planetary-mass members of the $\beta$ Pictoris moving group. Combining our $v \sin (i)$ measurement with recent photometric variability data, we constrain the inclination of PSO J318.5-22 to $>29^{\circ}$ and its rotational period to $5-10.2$ hours. The equatorial velocity of PSO J318.5-22 indicates that its rotation is consistent with an extrapolation of the velocitymass relationship for solar system planets.

Subject headings: brown dwarfs -infrared:stars - planets and satellites: atmospheres - techniques: radial velocities - techniques: spectroscopic - stars: rotation
\end{abstract}

\section{INTRODUCTION}

Objects with masses below the hydrogen burning limit $\left(\sim 75 M_{\text {Jup }}\right.$; Burrows et al. 2001) cool as they age, resulting in a mass-age-luminosity degeneracy. Several-Gyrold brown dwarfs can have the same luminosities as much younger, several-Myr-old planetary-mass $\left(\lesssim 13 M_{\text {Jup }}\right)$ objects. Thus, to determine the mass of a single substellar object, one must have an independent constraint on its age.

As planetary-mass objects and brown dwarfs cool, they also contract, increasing their surface gravity as they evolve. Thus evidence of low gravity (e.g. weak FeH and alkali features, strong VO absorption) in a spectrum can corroborate the youth of an object. As noted in Allers \& Liu (2013), however, spectroscopic ages are not particularly accurate, and objects of the same age and spectral type can have different indicators of youth.

Another method to determine the age of a substellar object is via association with a young stellar moving group. These groups have ages known from the stellar members of the groups, either from model isochrone fitting of the members on an HR diagram (e.g. Zuckerman et al. 2001; Pecaut et al. 2012) or the loca- tion of the lithium depletion boundaries (e.g. King et al. 2000; Binks \& Jeffries 2014). Though the estimated age of an individual group may vary by up to a factor of $\sim 2$ depending on the method of age determination, the various methods agree on the relative ages of the groups (e.g. Mentuch et al. 2008; da Silva et al. 2009; Bell et al. 2015). In order to link an object to a local young moving group, its galactic kinematics $(U V W)$ and position $(X Y Z)$ must agree with the other known members of the group.

PSO J318.5338-22.8603 (hereinafter PSO J318.5-22), discovered by Liu et al. (2013), is the first free-floating object with colors and luminosity that overlap the young, dusty, planetary-mass companions to HR 8799 and 2MASS J1207-39. Based on its parallax, proper motion, and spectroscopic signatures of youth (low-gravity), Liu et al. identified PSO J318.5-22 as a candidate member of the $\beta$ Pictoris (hereinafter $\beta$ Pic) moving group. Liu et al. estimate a mass of $6.5_{-1.0}^{+1.3} M_{\text {Jup }}$ for PSO J318.5-22, based on evolutionary models for an isochronal age of $12_{-4}^{+8} \mathrm{Myr}$ and the luminosity of PSO J318.5-22. Here, we report a determination of the radial and rotational velocities of PSO J318.5-22 for the 
first time and assess the probability that PSO J318.5-22 is a member of the $\beta$ Pic moving group. We re-estimate the physical properties of PSO J318.5-22 based on recent determinations of the age of the $\beta$ Pic moving group (23 \pm 3 Myr; Mamajek \& Bell 2014). With a mass and effective temperature intermediate to the directly-imaged exoplanets $\beta$ Pictoris b (Lagrange et al. 2009) and 51 Eridani b (Macintosh et al. 2015), PSO J318.5-22 is an important free-floating analog to these young planets. In addition, combining our measurement of $v \sin (i)$ with the recent measurements of PSO J318.5-22's orbital period (Biller et al. 2015), we constrain its spin axis inclination and equatorial velocity.

\section{OBSERVATIONS AND DATA REDUCTION}

We obtained a high resolution $(\mathrm{R} \approx 14000)$ spectrum of PSO J318.5-22 using the GNIRS spectrograph (Elias et al. 2006) on the Gemini-North Telescope. We used the 111 lines $\mathrm{mm}^{-1}$ grating with a 0.15 " slit, resulting in a $2.27-2.33 \mu \mathrm{m}$ spectrum. Our queue observations were obtained on UT 26 November 2013 under clear skies while the seeing was 0.49 ". Seven exposures of $600 \mathrm{~s}$ were obtained, nodding $6^{\prime \prime}$ along the slit between exposures for a total integration time of 70 minutes. Immediately after our observations of PSO J318.5-22, we obtained flat field and Xenon-Argon lamp images. We also observed an A0 star, HIP 110746, as a telluric standard using the same instrument setup.

Data were reduced using the REDSPEC reduction package to spatially and spectrally rectify each exposure, referencing the Keck/NIRSPEC Echelle Arc Lamp Tool (http://www2.keck.hawaii.edu/inst/nirspec/ N7arclamps.html) to identify the wavelengths of lines in our arc lamp spectrum. After nod-subtracting pairs of exposures, we create a spatial profile which is the median intensity across all wavelengths at each position along the slit. To remove any residual sky emission lines from our nod-subtracted pairs and to determine the noise per pixel at each wavelength, we identify pixels in the spatial profile that do not contain significant source flux. At each wavelength, we fit a line to the intensity of these background pixels vs. position along the slit. The fitted line provides the background level as a function of position and the scatter of the background pixels about the line gives the noise per pixel 1 We then performed a profile-weighted extraction (Horne 1986) on each exposure and combined the resulting spectra using a robust weighted mean with the xcombspec procedure from the SpeXtool package (Cushing et al. 2004). The median signal-to-noise of our final spectrum is 21.

We determine the FWHM of the line spread function (LSF) by fitting 1-dimensional gaussians to the Xenon and Argon lines in an extracted spectrum of the arc lamp image. For the seven arc lamp lines, we calculate a mean and standard deviation for the LSF FWHM of of $0.000163 \pm 0.000015 \mu \mathrm{m}$, corresponding to a resolution, $R=\lambda / \Delta \lambda=14100$.

\section{DETERMINING RADIAL AND ROTATIONAL VELOCITIES}

\footnotetext{
1 This assumes that the shot noise is dominated by the background. PSO J318.5-22 is faint, so the sky background level is $>3$ times brighter than the flux of our source across all wavelengths of our spectrum
}

Unfortunately, no radial velocity standard was observed as a part of our Gemini program. We use an approach similar to that described by Blake et al. (2010), wherein we employ forward modeling to simultaneously fit the wavelength solution of our spectrum, the rotational and radial velocities of PSO J318.5-22, the scaling of telluric line depths, and the FWHM of the instrumental LSF.

\subsection{Creating a Forward Model}

Our observed spectrum of PSO J318.5-22 is the combination of the spectrum of the brown dwarf, telluric absorption, and effects from the instrument (wavelengthdependent response and line spread functions). We create myriad model spectra and evaluate the best fit parameters by comparison to our observed spectrum of PSO J318.5-22. The components of our forward model are:

- Wavelength Solution: When reducing our data, the wavelength solution of our observed spectrum is determined from seven Xenon-Argon arc lines with wavelengths of $2.29-2.33 \mu \mathrm{m}$. There are no arc lines for the $2.27-2.29 \mu \mathrm{m}$ portion of our spectrum. In addition, the typical residuals to our wavelength solution for each arc line correspond to $\sim 5 \mathrm{~km} \mathrm{~s}^{-1}$. Our spectrum itself contains many telluric absorption features which allow us to refine the wavelength solution as a part of our forward modeling. We calculate the wavelength solution of our observed spectrum using a 2nd-degree polynomial. Rather than having the coefficients of the polynomial as our fitted parameters, we use barycentric Lagrangian interpolation (Berrut \& Trefethen 2004; Waring 1779), solving for the wavelengths of the first, middle, and last pixels of the spectrum and determining the coefficients of the unique 2nddegree polynomial that will fit these three points. We start by adopting the wavelengths of our reduced spectrum as the initial guess.

- Brown Dwarf Atmosphere Model: We use the BT-Settl model atmospheres (Allard et al. 2012) as the intrinsic spectrum of PSO J318.5-22 in our forward modeling. The BT-Settl models are provided for two different assumed solar abundances (Asplund et al. 2009 [AGSS] and Caffau et al. 2011 [CIFIST]). We use model grids with effective temperatures $\left(T_{e f f}\right)$ of $1100-2500 \mathrm{~K}$ and surface gravities $(\log (g))$ of 3.5-5.5 dex (where $g$ is in units of $\mathrm{cm} \mathrm{s}^{-2}$.) We adjust the wavelengths of the model atmosphere to account for the radial velocity of the object, $\lambda=\lambda_{0}+\left(\left(v_{r}+v_{\text {bary }}\right) \lambda_{0} / c\right)$, where $v_{r}$ is the radial velocity of PSO J318.5-22, and vary $\left(28.1 \mathrm{~km} \mathrm{~s}^{-1}\right)$ is the barycentric motion projected away from PSO J318.5-22 at the time of our observation. We then convolve the model spectrum to account for rotation, $v \sin (i)$, using a convolution kernel created by the LSF_ROTATE task 2 . Our velocity-shifted, rotationally broadened model atmosphere approximates the spectrum of the brown dwarf itself.

2 A part of the IDL Astronomy User's Library 
- Telluric Absorption: We use the telluric spectrum of Livingston \& Wallace (1991)3. We adjust the depths of the telluric features and create a new telluric spectrum, $T(\lambda)=T_{0}(\lambda)^{\tau}$, where $T_{0}(\lambda)$ is the Livingston \& Wallace (1991) spectrum, and $\tau$ is one of the parameters of our fit. The velocity-shifted, rotationally broadened brown dwarf model atmosphere is then multiplied by the telluric spectrum, approximating the spectrum of PSO J318.5-22 as it enters the telescope.

- Instrumental Effects: We approximate the instrumental LSF as a gaussian and fit for the FWHM of the gaussian convolution kernel. We convolve the brown dwarf model atmosphere with telluric absorption by this gaussian kernel. We account for any changes in spectral shape between our model and the observed spectrum by taking the ratio of our observed spectrum of PSO J318.5-22 to our model spectrum, fitting the smoothed ratio with a 3rd order polynomial, multiplying our model by the fit, and then scaling our model to the median of our observed spectrum.

In total, our forward model has nine free parameters: the $T_{\text {eff }}$ and $\log (g)$ of the atmospheric model, the $v_{r}$ and $v \sin (i)$ of PSO J318.5-22, $\tau$ for the telluric spectrum, the LSF FWHM, and the wavelengths of the first, middle and last pixels. The forward model is compared to our observed spectrum, and the parameters used to create the forward model are adjusted to achieve the best fit.

\subsection{Parameter Optimization with AMOEBA}

AMOEBA uses a downhill simplex method (Nelder \& Mead 1965) to find the minimum of a multivariate function. We used IDL's implementation of AMOEBA, which is based on Numerical Recipes in $C$ (Press et al. 2007). We run our AMOEBA optimization for each model atmosphere having $T_{\text {eff }}=900-2500 \mathrm{~K}$ and $\log (g)=3.5-5.5$ in the CIFIST and AGSS grids. AMOEBA does not conduct a thorough search of parameter space, so it is important to seed it with reasonable parameter estimates. We initialize AMOEBA with $v_{r}=0 \mathrm{~km} \mathrm{~s}^{-1}$ and $v \sin (i)=15 \mathrm{~km} \mathrm{~s}^{-1}$, typical of observed values of ultracool dwarfs (Blake et al. 2010). We use the FWHM of the LSF as determined from our arc lamp spectrum $(\S 2)$ as a starting point. To get a starting point for $\tau$, we divide our observed telluric standard spectrum by an LSF-convolved telluric absorption spectrum and find the value of $\tau(0.7)$ that, by eye, results in a featureless spectrum (as expected for an A0 star from $2.27-2.33 \mu \mathrm{m})$.

We find that the atmospheric model giving the best overall fit (minimum $\chi^{2}$ ), is the CIFIST model having $T_{\text {eff }}=1650 \mathrm{~K}$ and $\log (g)=5.0$, in reasonable agreement with the temperature $(1400-1600 \mathrm{~K})$ and $\log (\mathrm{g})(4.0-4.5)$ of the atmospheric models which fit the low-resolution $0.8-2.5 \mu \mathrm{m}$ spectrum of PSO J318.5-22 (Liu et al. 2013). The best fit parameters returned for this model are: FWHM of $\mathrm{LSF}=0.000169 \mu \mathrm{m}, \tau=0.868$, $v \sin (i)=11.6 \mathrm{~km} \mathrm{~s}^{-1}$, and $v_{r}=-7.4 \mathrm{~km} \mathrm{~s}^{-1}$.

3 available for download at ftp://ftp.noao.edu/catalogs/ atmospheric_transmission/transdata_1_5_mic.gz

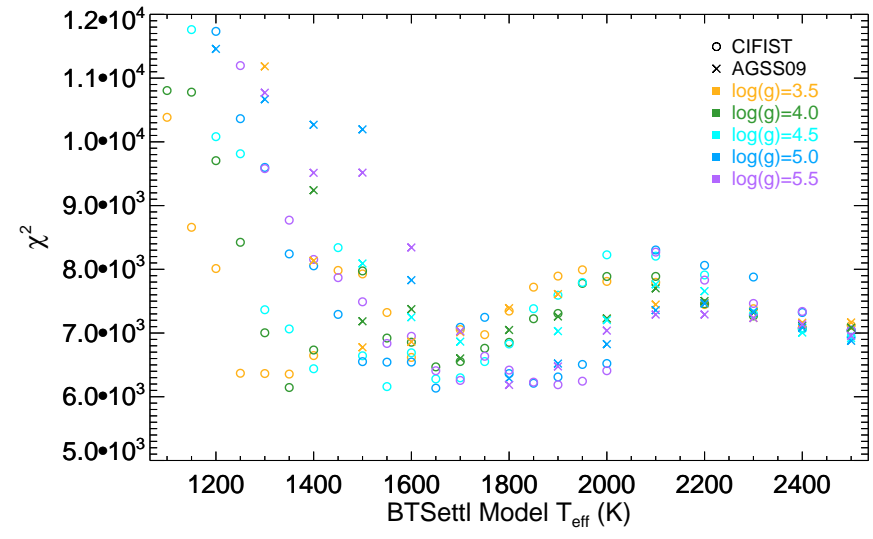

Figure 1. AMOEBA's best fit $\chi^{2}$ using BT-Settl models. The minimum $\chi^{2}$ occurs for the CIFIST model with $T_{e f f}=1650 \mathrm{~K}$ and $\log (g)=5.0$. In general, the CIFIST models provide marginally better fits than the AGSS09 models.

Overall, the CIFIST models provide better fits than the AGSS models (Figure 1). Regardless of the assumed solar abundance, $T_{\text {eff }}$, or $\log (g)$ of the atmospheric model, we find that AMOEBA converges on a radial velocity of -7.4 to $-2.3 \mathrm{~km} \mathrm{~s}^{-1}$. In contrast, the $v \sin (i)$ that AMOEBA finds varies significantly (0 to $80 \mathrm{~km} \mathrm{~s}^{-1}$ ) depending on the model used. We find that the fitted $v \sin (i)$ and resulting $\chi^{2}$ of the model fit are correlated. For atmospheric models with fits having higher $\chi^{2}$ values, AMOEBA converges on higher values of $v \sin (i)$. From this analysis, it is unknown if the higher $\chi^{2}$ values mean the $v \sin (i)$ of PSO J318.5-22 is genuinely low, or if for atmospheric models that do not approximate the spectrum of PSO J318.5-22 a higher $v \sin (i)$ smears out the lines and results in a better fit.

Our AMOEBA fitting provides a useful first estimate of the best fit parameters but lacks a straightforward way to determine their uncertainties. In addition, the correlation between $\chi^{2}$ and $v \sin (i)$ motivates a more sophisticated analysis to determine our best fit parameters and their associated uncertainties.

\subsection{Parameter Optimization with $M C M C$ and $\operatorname{DREAM}(Z S)$}

To better determine the best fit parameters of our forward model as well as their marginalized posterior distributions, we use a Markov Chain Monte Carlo (MCMC) approach. We create forward models in the manner described above but in addition allow for a continuous distribution of $T_{\text {eff } f}$ and $\log (g)$ by linearly interpolating between atmosphere models in the CIFIST grid. We employ the DREAM(ZS) algorithm of ter Braak \& Vrugt (2008), which involves an adaptive stepper, updating model parameters based on chain histories. A brief description of the algorithm follows.

Three chains are run in parallel, and each chain is a history of accepted parameter vectors p. Current values of the 3 chain vectors are stored in the array $\mathbf{X}$, and chain histories are stored in the array $\mathbf{Z}$. To simplify the notation, $\mathbf{X}[i]$ refers to the $n$-dimensional, $i$-th parameter vector currently stored in $\mathbf{X}$ (i.e., the $i$-th chain in $\mathbf{X}$ ), and we adopt the same notation for the history array, $\mathbf{Z}[i]$. We initially seed each chain with the parameters of the best-fit model as determined by our AMOEBA analysis $(\S 3.2)$. 


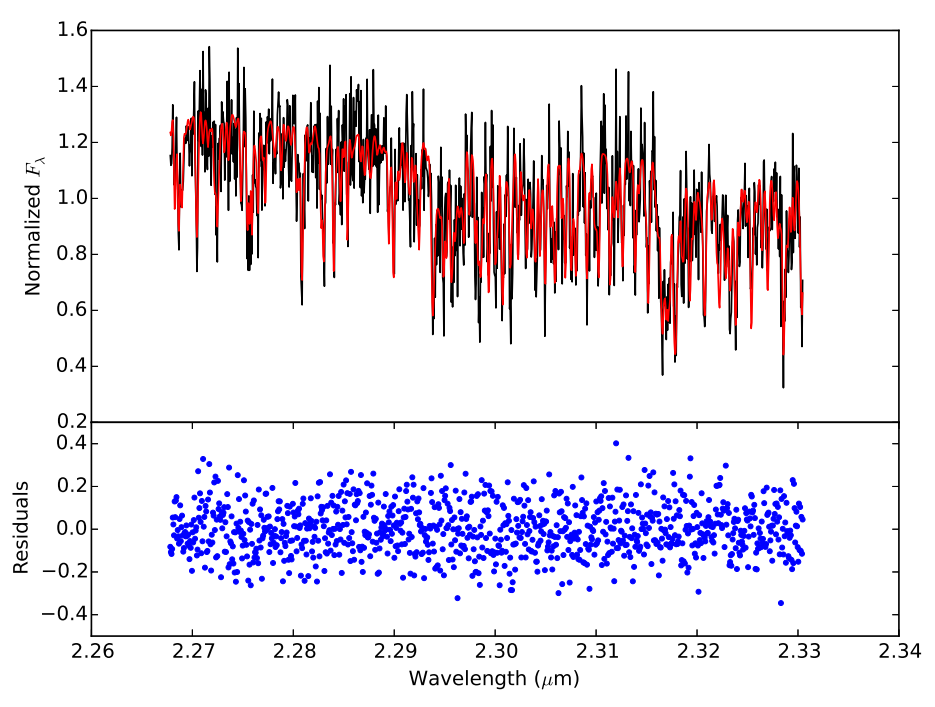

Figure 2. Our observed spectrum of PSO J318.5-22 (black) compared to our the forward model with our best fit parameters (red). The median residual of the fit is 0.079 (in normalized $F_{\lambda}$ units), which is larger than the median uncertainty (0.048) of our observed spectrum. The rms of the residuals (0.12) indicates systematic uncertainties of $\approx 10 \%$.

At each iteration and for each chain $\mathbf{X}[i]$, two parameter vectors $\mathbf{Z}[j]$ and $\mathbf{Z}[k]$ are selected at random and without replacement from $\mathbf{Z}$. A trial parameter vector, $\mathbf{x}$, is then calculated by the difference,

$$
\mathbf{x}[i]=\mathbf{X}[i]+(1+\mathbf{e}) \gamma(\mathbf{Z}[j]-\mathbf{Z}[k])+\epsilon,
$$

where $\gamma$ is a constant of order unity, $\mathbf{e}$ is an $n$-dimensional vector of uniformly-distributed random numbers spanning $[-0.05,0.05]$, and $\epsilon$ is a vector of normallydistributed random numbers with zero mean and $\sigma=$ $10^{-6}$.

The trial vector is accepted or rejected using the standard Metropolis-Hastings likelihood ratio criterion (Metropolis et al. 1953; Hastings 1970). We assume normally distributed uncertainties. Therefore, the loglikelihood of a parameter vector is given by $\ln (\mathbf{p})=$ $C-\chi^{2} / 2$, where $C$ is a normalization constant that cancels out in the likelihood ratio, and $\chi^{2}$ is the usual chisquared statistic.

Every ten iterations (the thinning interval), the current chain values $\mathbf{X}$ are appended to the history array $\mathbf{Z}$. Note that once the chains are appended to $\mathbf{Z}$, they lose identity, so that the difference vectors that determine the step for any one chain depends on the history of all three chains. This property allows any chain in $\mathbf{X}$ to hop between modes in the posterior distributions of the parameters. To search for modes and allow their inclusion in the parameter distribution, the algorithm sets $\gamma=1$ every ten iterations.

The DREAM(ZS) algorithm also employs a more complicated "snooker" updater that relies instead on three draws from Z. ter Braak \& Vrugt (2008) demonstrated that the snooker updater complements the difference updater described above, arguably allowing a more efficient search of parameter space than would be provided by difference steps alone. In our implementation, there is a $10 \%$ chance of a given iteration using snooker rather than difference updates.
We run $10^{5}$ iterations, which, taking into account three chains and ten steps per interval, corresponds to $3 \times 10^{6}$ functional evaluations of the model. Parameter distributions and summary statistics are derived from the final state of the history array $\mathbf{Z}$. Since we initialize $\mathbf{Z}$ using random draws from the parameter support regions, the early values of $\mathbf{Z}$ are drawn far from the equilibrium parameter distribution and represent expectedly poor fits to the data. These initial "burn-in" values are identified based on an evaluation of $\chi^{2}$ values, and they are discarded before analysis of the chains. Formally, we divide the $\chi^{2}$ vector into 20 equal intervals. The boundary between non-equilibrium and equilibrium intervals is determined using the Geweke (1992) diagnostic to evaluate the difference of means between a given interval and the final interval.

In our earlier AMOEBA analysis, we found that the uncertainties in our measured spectrum were systematically smaller than the residuals of the fit. Adding in an additional $\sim 10 \%$ uncertainty brings the residuals and uncertainties into reasonable agreement. This discrepancy could be due to underestimated uncertainties in our observed spectrum and/or systematic uncertainties in our telluric spectrum and the brown dwarf model atmospheres. To account for the effect of these systematic uncertainties in the posterior distributions of our MCMC analysis, we include a uniform $10 \%$ systematic uncertainty which is added in quadrature to the measurement errors of our observed spectrum.

Our spectrum of PSO J318.5-22 as well as the best fit model is displayed in Figure 2. The best fit model has $T_{\text {eff }}=1325_{-12}^{+330} \mathrm{~K}$ and $\log (g)=3.7_{-0.1}^{+1.1}$ dex. We determine a best fit LSF FWHM of $0.00016 \pm 0.00001 \mu \mathrm{m}$, in agreement with the LSF FWHM from our arc lamp spectrum $(0.00016 \pm 0.00002 \mu \mathrm{m})$. The reduced $\chi^{2}$ for our best fit model is 1.24 . Figure 3 shows the marginalized posterior distributions for $v_{r}$ and $v \sin (i)$. We find $v_{r}=-6.0_{-1.1}^{+0.8} \mathrm{~km} \mathrm{~s}^{-1}$ and $v \sin (i)=17.5_{-2.8}^{+2.3} \mathrm{~km} \mathrm{~s}^{-1}$ for PSO J318.5-22.

\section{MEMBERSHIP IN $\beta$ PICTORIS}

Based on its parallactic distance and proper motion, Liu et al. (2013) identified PSO J318.5-22 as a likely $\beta$ Pic member. The Bayesian analysis of Gagné et al. (2014) found a 99.7\% probability that PSO J318.5-22 is a $\beta$ Pic member and predicted a radial velocity of $-6.4 \pm 1.7 \mathrm{~km} \mathrm{~s}^{-1}$. To determine the full, threedimensional velocity and position of PSO J318.5-22, we combine our $v_{r}$ measurement with the updated parallax and proper motion from Liu et al. (2015, in preparation): $\pi=0$.'0450 $\pi$ 0.'0017; $\mu_{\alpha} \cos \delta=$

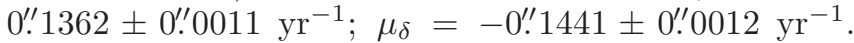
Using the three-dimensional velocity and position of PSO J318.5-22, we recalculated membership probabilities using the BANYAN-II web too 5 (Gagné et al. 2014; Malo et al. 2013) and find that the probability of membership in $\beta$ Pic increases to $99.98 \%$. Thus, our measured radial velocity is consistent with membership in $\beta$ Pic.

We determine galactic $X Y Z$ position and $U V W$ ve-

4 Because AMOEBA does not propagate uncertainties, including a systematic $10 \%$ uncertainty reduces the $\chi^{2}$ of our AMOEBA fits, but does not affect the best fit parameters.

5 http://www . astro.umontreal.ca/ gagne/banyanII.php 

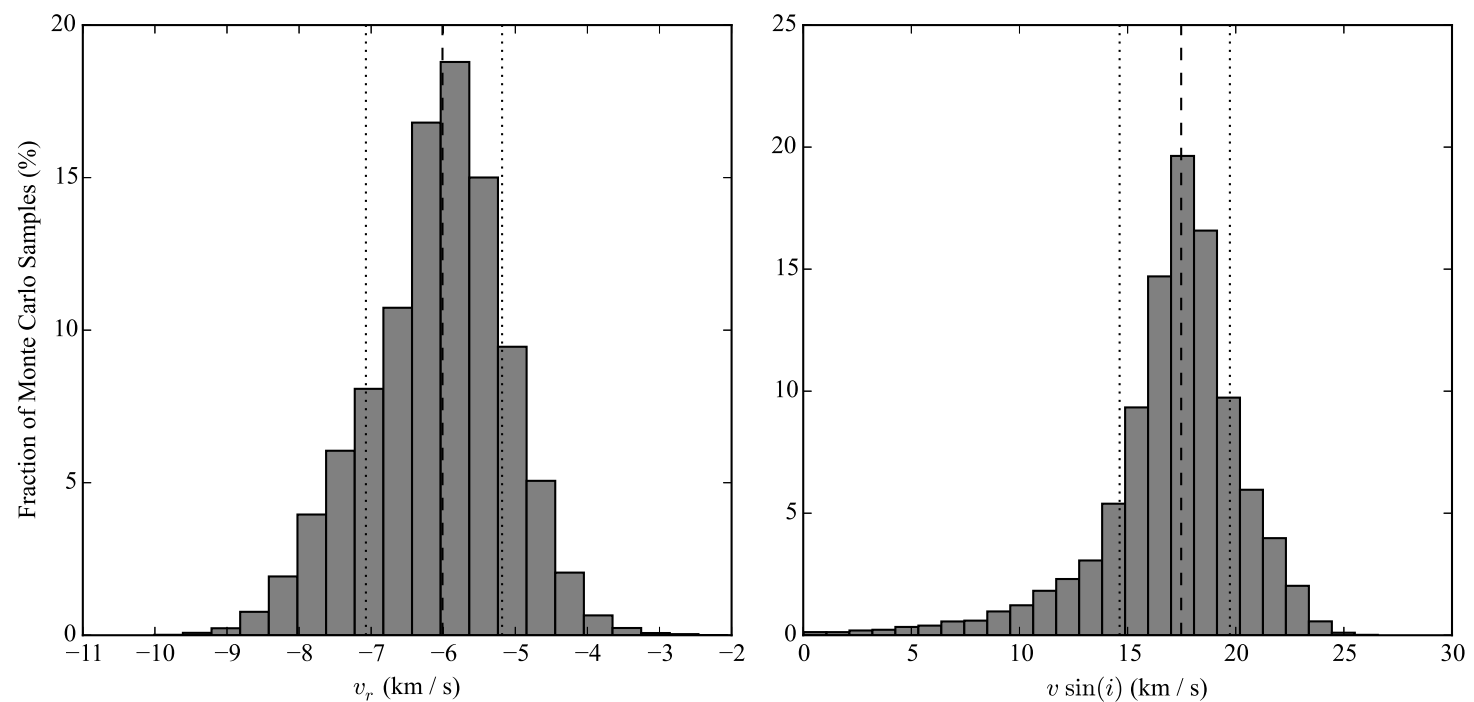

Figure 3. Histograms of the marginalized posterior distributions for $v_{r}$ and $v \sin (i)$ from our MCMC analysis. The dashed lines indicate the median of each distribution. The dotted lines indicate the $68 \%$ confidence limits $(1-\sigma)$ centered on the median.

Table 1

Galactic Positions and Velocities

\begin{tabular}{lrrrrrr}
\hline \hline \multicolumn{1}{c}{ Name } & \multicolumn{1}{c}{$\mathrm{X}$} & \multicolumn{1}{c}{$\mathrm{Y}$} & \multicolumn{1}{c}{$\mathrm{Z}$} & \multicolumn{1}{c}{$\mathrm{U}$} & \multicolumn{1}{c}{$\mathrm{V}$} & \multicolumn{1}{c}{$\mathrm{W}$} \\
$\cdots$ & $\mathrm{pc}$ & \multicolumn{1}{c}{$\mathrm{pc}$} & \multicolumn{1}{c}{$\mathrm{pc}$} & \multicolumn{1}{c}{$\mathrm{km} \mathrm{s}^{-1}$} & \multicolumn{1}{c}{$\mathrm{km} \mathrm{s}^{-1}$} & \multicolumn{1}{c}{$\mathrm{km} \mathrm{s}^{-1}$} \\
\hline$\beta$ Pic Group $^{\mathrm{a}}$ & $8.4 \pm 31.9$ & $-5.0 \pm 15.4$ & $-15.0 \pm 8.0$ & $-10.9 \pm 1.5$ & $-16.0 \pm 1.4$ & $-9.2 \pm 1.8$ \\
PSO J318.5-22 & $15.2 \pm 0.6$ & $7.2 \pm 0.3$ & $-14.6 \pm 0.6$ & $-10.4 \pm 0.7$ & $-16.4 \pm 0.6$ & $-9.8 \pm 0.8$ \\
Offset & $6.7 \pm 31.8$ & $12.2 \pm 15.4$ & $0.4 \pm 8.0$ & $0.5 \pm 1.6$ & $-0.4 \pm 1.7$ & $-0.6 \pm 2.0$ \\
\hline
\end{tabular}

a From Mamaiek \& Bell (2014). The uncertainties in $U V W$ include both uncertainties in the mean $U V W$ for the group as well as the $1 \sigma$ dispersion of the group members.

locities for PSO J318.5-22 using its parallax and proper motions and our radial velocity measurement. We use a Monte Carlo approach with 10000 iterations to determine uncertainties in our calculated parameters $(U V W X Y Z)$ based on the uncertainties in our measured quantities. Table 1 includes the $X Y Z$ positions and $U V W$ velocities for both PSO J318.5-22 and the $\beta$ Pic group (Mamajek \& Bell 2014). As shown in Table 11 the difference in the galactic position and velocity between PSO J318.5-22 and the $\beta$ Pic group are well within the uncertainties. The total velocity of PSO J318.5-22 is $0.8 \pm 1.8 \mathrm{~km} \mathrm{~s}^{-1}$ from the mean velocity of the $\beta$ Pic group. The reduced $\chi^{2}$ for membership based on $U V W X Y Z$ and associated uncertainties (Table 11) for PSO J318.5-22 and the $\beta$ Pic group is a mere 0.15. For comparison, we calculated the velocity offset from the mean velocity of the $\beta$ Pic group for each of the stars listed as "bona fide" $\beta$ Pic moving group members in Gagné et al. (2014) 6 . We find that PSO J318.5-22 is a closer match to the mean kinematics of the $\beta$ Pic group than 46 out of 50 of the Gagné et al. "bona fide" members. Figure 4 shows the galactic position and velocities of PSO J318.5-22 compared to young moving groups from Torres et al. (2008). Regardless of which list of known members is used for comparison (Mamajek \& Bell 2014; Gagné et al. 2014; Torres et al. 2008), PSO J318.5-22 is determined to be a member of

\footnotetext{
${ }^{6}$ https://jgagneastro.wordpress.com/banyanii/
}

the $\beta$ Pic group.

\section{THE PHYSICAL PROPERTIES OF PSO J318.5-22}

Liu et al. (2013) estimated the mass of PSO J318.5-22 based on an age of $12_{-4}^{+8}$ Myr for $\beta$ Pic. Recently, revised age estimates indicate that the group is older. Mamajek \& Bell (2014) determined an age of $23 \pm$ $3 \mathrm{Myr}$ by combining isochronal ages Mamajek \& Bell 2014; Malo et al. 2014) and lithium depletion boundary ages (Binks \& Jeffries 2014; Malo et al. 2014). For a uniformly-distributed age of $23 \pm 3 \mathrm{Myr}$ and a normallydistributed luminosity of $\log \left(\mathrm{L}_{\mathrm{bol}} / \mathrm{L}_{\odot}\right)=-4.52 \pm$ 0.04 dex, we determine the mass, $T_{e f f}$, radius and $\log (g)$ of PSO J318.5-22 using model isochrones (Table 2). In general, the parameters determined from all four sets of models agree quite well. The cloud-free evolutionary models (Ames-COND and Saumon \& Marlev cloudless) yield slightly lower masses and smaller radii than models with clouds (Ames-DUSTY and Saumon \& Marley $f_{\text {sed }}=2$ ). PSO J318.5-22 has incredibly red $J-K$ colors, indicating a very cloudy atmosphere (Liu et al. 2013). The Ames-DUSTY models are not recommended for use with objects having $T_{\text {eff }}$ cooler than $1700 \mathrm{~K}$ (Allard et al. 2001). Thus, we adopt the physical properties as determined by the Saumon \& Marley $f_{\text {sed }}=$ 2 models: mass $=8.3 \pm 0.5 M_{\mathrm{Jup}}, T_{\text {eff }}=1127_{-26}^{+24} \mathrm{~K}$, radius $=1.464_{-0.008}^{+0.009} R_{\text {Jup }}$, and $\log (g)=4.01 \pm 0.03$ dex.

The $T_{\text {eff }}$ determined by forward-modeling our spectrum, $1325_{-12}^{+330} \mathrm{~K}$, is significantly higher than the $T_{\text {eff }}$ 

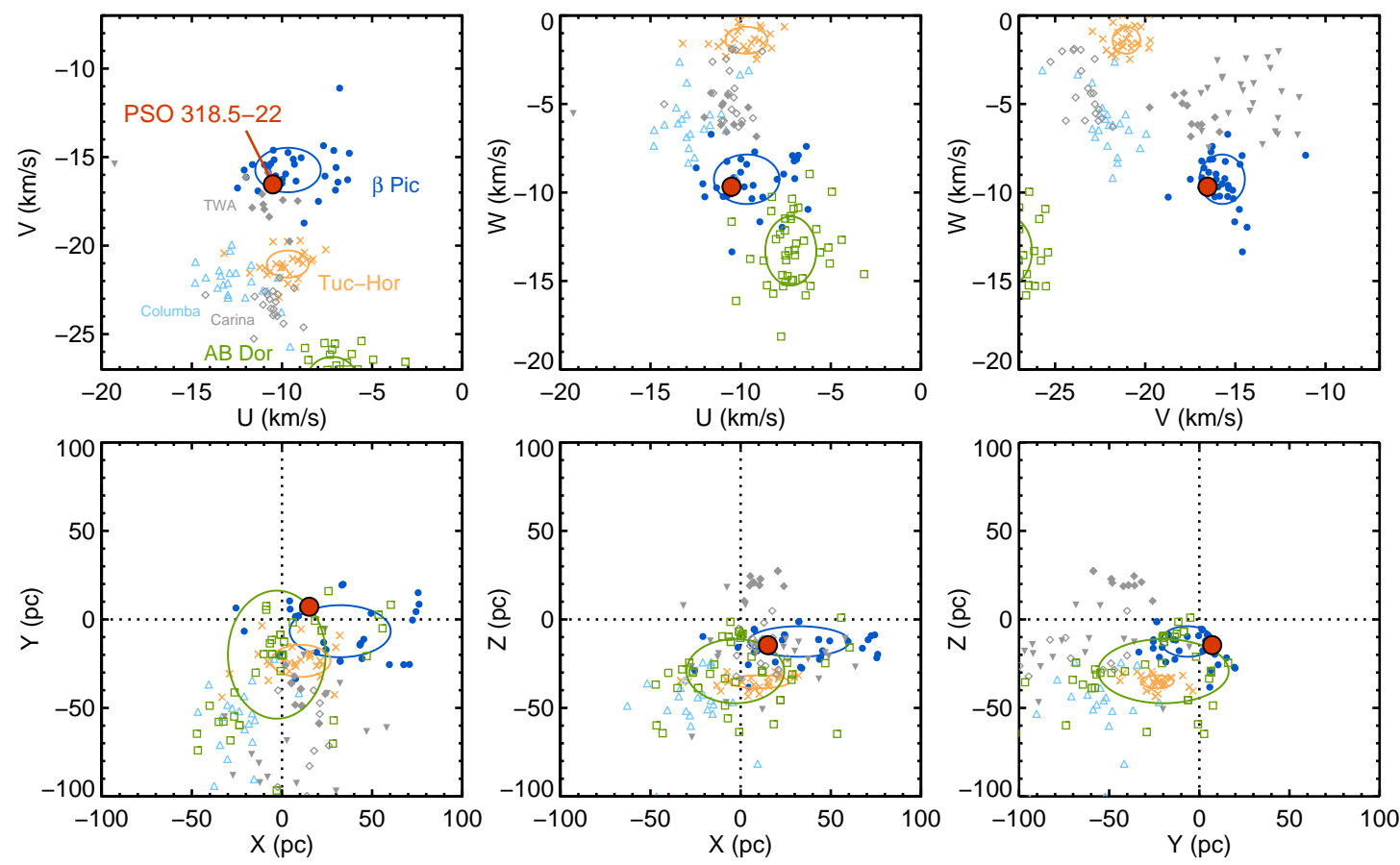

Figure 4. Comparison of the galactic position and velocity of PSO J318.5-22 to members of known young moving groups from Torres et al. (2008). Ellipses represent the rms for members of the group. The uncertainties in $U V W$ and $X Y Z$ for PSO J318.5-22 are smaller than the plotted red circle.

Table 2

Physical Properties from Evolutionary Models

\begin{tabular}{|c|c|c|c|c|c|}
\hline $\begin{array}{l}\text { Evolutionary Model } \\
\text { Name }\end{array}$ & Ref & $\begin{array}{l}\text { Mass } \\
M_{\text {Jup }}\end{array}$ & $\begin{array}{c}T_{e f f} \\
\mathrm{~K}\end{array}$ & $\begin{array}{c}\text { Radius } \\
R_{\text {Jup }}\end{array}$ & $\begin{array}{c}\log (g) \\
\operatorname{dex}\end{array}$ \\
\hline \multicolumn{6}{|c|}{ PSO J318.5-22 $\left(\log \left(\mathrm{L}_{\mathrm{bol}} / \mathrm{L}_{\odot}\right)=-4.52 \pm 0.04 \mathrm{dex}^{5} ;\right.$ age $\left.=23 \pm 3 \mathrm{Myr}^{7}\right)$} \\
\hline AMES-COND & 1 & $7.9 \pm 0.4$ & $1176_{-25}^{+26}$ & $1.358 \pm 0.010$ & $4.03 \pm 0.03$ \\
\hline AMES-Dusty & 4 & $8.7 \pm 0.4$ & $1154_{-27}^{+25}$ & $1.417 \pm 0.007$ & $4.00 \pm 0.02$ \\
\hline Saumon \& Marlev cloudless & 8 & $7.9 \pm 0.4$ & $1164_{-27}^{+26}$ & $1.373 \pm 0.010$ & $4.04 \pm 0.03$ \\
\hline Saumon \& Marley $f_{\text {sed }}=2$ & 8 & $8.3 \pm 0.5$ & $1127_{-26}^{+24}$ & $1.464 \pm 0.010$ & $4.01 \pm 0.03$ \\
\hline \multicolumn{6}{|c|}{$\beta$ Pic b $\left(\log \left(\mathrm{L}_{\mathrm{bol}} / \mathrm{L}_{\odot}\right)=-3.86 \pm 0.04 \mathrm{dex}^{6} ;\right.$ age $\left.=23 \pm 3 \mathrm{Myr}^{7}\right):$} \\
\hline Saumon \& Marley $f_{\text {sed }}=2$ & 8 & $12.8 \pm 0.2$ & $1583_{-33}^{+30}$ & $\overline{1.576} \pm 0.010$ & $4.135 \pm 0.003$ \\
\hline \multicolumn{6}{|c|}{$\underline{2 \mathrm{M} 1207 \mathrm{~b}\left(\log \left(\mathrm{L}_{\mathrm{bol}} / \mathrm{L}_{\odot}\right)=-4.68 \pm 0.05 \mathrm{dex}^{2} ; \text { age }=10 \pm 3 \mathrm{Myr}^{3}\right)}$} \\
\hline Saumon \& Marlev $f_{\text {sed }}=2$ & 8 & $4.5 \pm 0.5$ & $1006_{-26}^{+24}$ & $1.515_{-0.013}^{+0.016}$ & $3.71 \pm 0.06$ \\
\hline
\end{tabular}

inferred by evolutionary models. This discrepancy is similar to the result found by Liu et al. (2013) using a much lower resolution spectrum spanning a larger wavelength range. This hints that atmospheric models over-predict $T_{\text {eff }}$ when fitting either the broad-band spectral morphology seen in low-resolution spectra or the depths of spectral lines seen in high resolution spectra.

The mass we determine for PSO J318.5-22 is somewhat higher than the $6.5_{-1.0}^{+1.3} M_{\text {Jup }}$ reported by Liu et al.
(2013). This can be attributed to the revised (older) age of the $\beta$ Pic moving group. For comparison to PSO J318.5-22, we calculate the properties of $\beta$ Pic b and 2M1207b (Table 2) using the Saumon \& Marley $f_{\text {sed }}=2$ evolutionary models and accounting for recent revisions to the ages of the $\beta$ Pic and TW Hydra moving groups. The mass of PSO J318.5-22 (8.3 \pm $\left.0.5 M_{\mathrm{Jup}}\right)$ is intermediate to the two known directlyimaged exoplanets around $\beta$ Pic members, $\beta$ Pic b and 


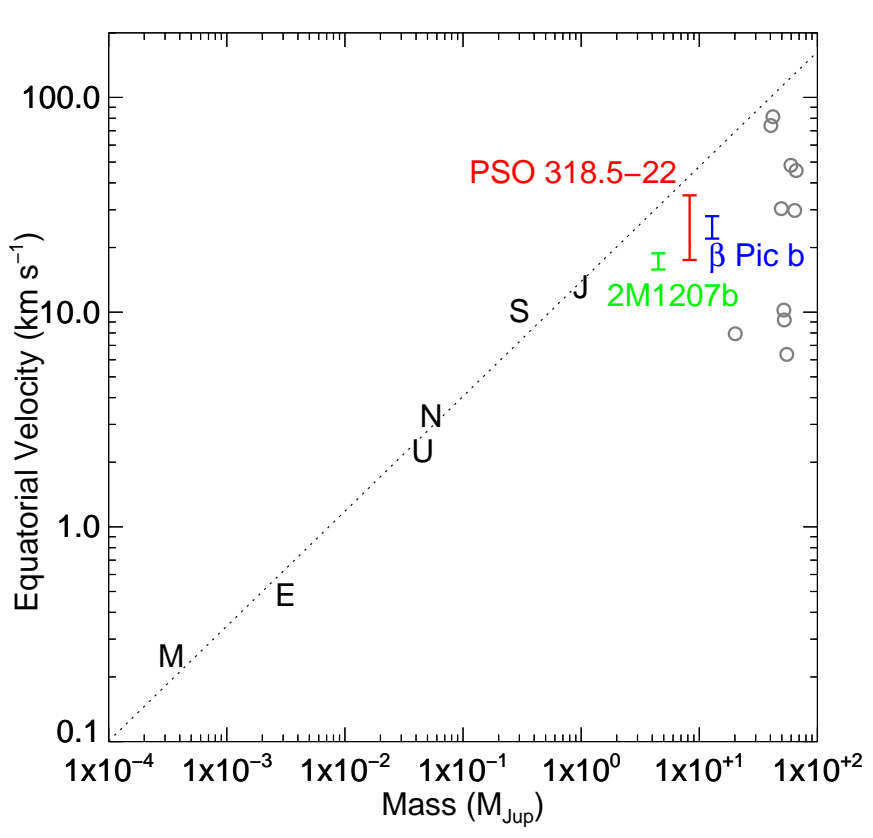

Figure 5. A variation on Figure 2 of Snellen et al. (2014) comparing the equatorial velocities of planets and brown dwarfs. The red bar indicates our constraints on the equatorial velocity of PSO J318.5-22. The blue data point shows the equatorial velocity (Snellen et al. 2014) and estimated mass (see §5) of $\beta$ Pic b. The green data point shows the equatorial velocity of $2 \mathrm{M} 1207 \mathrm{~b}$ calculated from its rotation period (Zhou et al. 2015) and estimated radius (Table 2). The gray open circles indicate the equatorial velocities of field brown dwarfs calculated from period measurements in Metchev et al. (2015) and mass and radius estimates from Filippazzo et al. (2015). Solar system objects are plotted as the first letter of their name. Mercury and Venus are excluded due to their extremely low equatorial velocities. The dotted line shows a linear fit to $\log$ (mass) vs. $\log$ (velocity) for the solar system planets plotted.

51 Eri b ( $\sim 2 M_{\text {Jup }}$; Macintosh et al. 2015). In addition, PSO J318.5-22 is an excellent free-floating analog to $\beta$ Pic b and 2M1207b, the only other planetary-mass objects having constraints on their rotation.

\section{CONSTRAINING THE ROTATION AND INCLINATION OF PSO J318.5-22}

Our measurement of $v \sin (i)=17.5_{-2.8}^{+2.3} \mathrm{~km} \mathrm{~s}^{-1}$ allows us to constrain the equatorial velocity and inclination of PSO J318.5-22. Biller et al. (2015) report the detection of $J_{S^{-}}$-band variability, likely due to rotational modulation of inhomogeneous cloud cover. Unfortunately, the measurements of Biller et al. (2015) do not cover a long enough time span to measure a full rotational period of PSO J318.5-22, but they constrain the period to $\geq 5$ hours. Assuming a radius of PSO J318.5-22 of $1.464 R_{\mathrm{Jup}}$, a period of $>5$ hours corresponds to an equatorial velocity of $<36 \mathrm{~km} \mathrm{~s}^{-1}$. Thus, our $v \sin (i)$ measurement in combination with the Biller et al. result constrains the equatorial velocity to $17.5-36 \mathrm{~km} \mathrm{~s}^{-1}$ and the inclination of PSO J318.5-22 to $i \geq 29^{\circ}$. In addition, our $v \sin (i)$ measurement places an upper limit on the rotational period of 10.2 hours. A period of 5-10.2 hours is slightly longer than the typical $\sim 3$ hour periods of $\mathrm{L} / \mathrm{T}$ transition field brown dwarfs (Radigan et al. 2014), which is expected given that PSO J318.5-22 is young and still in the process of contracting.
Our constraints on its equatorial velocity (17.5$36 \mathrm{~km} \mathrm{~s}^{-1}$ ) allow us to compare the rotation of PSO J318.5-22 to recent measurements of the rotation of other planetary-mass and substellar objects. The equatorial velocity of $\beta$ Pic b, $25 \mathrm{~km} \mathrm{~s}^{-1}$ (Snellen et al. 2014), is consistent with our constraint on the equatorial velocity of PSO J318.5-22. The $10.7_{-0.8}^{+1.2}$ hour period of 2M1207b (Zhou et al. 2015) is slighty longer than our upper limit on the period for PSO J318.5-22. Given 2M1207b's lower mass and younger age (see $\S 5$ ), it is expected to rotate more slowly than $\beta$ Pic b and PSO J318.5-22. Indeed, the equatorial velocity of $2 \mathrm{M} 1207 \mathrm{~b}$ is $17.3 \pm 1.5 \mathrm{~km} \mathrm{~s}^{-1}$ as calculated from its rotational period and evolutionary-model inferred radius. The agreement in equatorial velocities for 2M1207b, PSO J318.5-22 and $\beta$ Pic b hints that the angular momentum evolution of free-floating and gravitationally-bound planetary mass objects is similar. For comparison, we calculate the equatorial velocities for field brown dwarfs with measured periods in Metchev et al. (2015) and with estimated masses and radii from Filippazzo et al. (2015). We find that field brown dwarfs have a relatively broad range of equatorial velocities $\sim 6-80 \mathrm{~km} \mathrm{~s}^{-1}$. Additional rotation measurements for planetary-mass objects are needed to determine if the field brown dwarf spread in equatorial velocity extends to lower masses. Figure 5 compares the equatorial velocity of PSO J318.5-22 to that of $\beta$ Pic b (Snellen et al. 2014), planets in our solar system[7, and field brown brown dwarfs. Solar system planets have a log-log velocity-mass relationship. 2M1207b, $\beta$ Pic $\mathrm{b}$ and PSO J318.5-22 are slower rotating than would be expected from the extrapolation of the solar system velocity-mass relationship. This is likely due to their young ages. By the time they are the age of our solar system, the equatorial velocities of $2 \mathrm{M} 1207 \mathrm{~b}, \beta$ Pic b, and PSO J318.5-22 should increase by a factor of $\sim 1.5$ as they contract to a radius of $\sim 1 R_{\mathrm{Jup}}$, putting them in good agreement with the velocity-mass relation seen for the solar system planets.

\section{CONCLUSIONS}

Using forward modeling techniques, we have measured the radial velocity $\left(-6.0_{-1.1}^{+0.8} \mathrm{~km} \mathrm{~s}^{-1}\right)$ and $v \sin (i)$ $\left(17.5_{-2.8}^{+2.3} \mathrm{~km} \mathrm{~s}^{-1}\right)$ of PSO J318.5-22. Combining our $v_{r}$ with an updated parallax and proper motion of PSO J318.5-22, we determine its $U V W$ velocities. We find that the galactic position and motion of PSO J318.5-22 are consistent with membership in the $\beta$ Pic moving group. The luminosity of PSO J318.5-22, combined with recent age determinations of the $\beta$ Pic moving group allow us to use evolutionary models to estimate its mass, $T_{\text {eff }}$, radius, and $\log (g)$. Using our $v \sin (i)$ measurement and recently published variability results, we determine that the inclination of PSO J318.5-22 must be greater than $29^{\circ}$ and the rotational period is constrained to $5-10.2$ hours. Our constraints on the equatorial velocity of PSO J318.5-22 indicate that its rotation is consistent with an extrapolation of the velocity-mass relationship of solar system planets.

\footnotetext{
7 http://nssdc.gsfc.nasa.gov/planetary/factsheet/
} 
With its well-determined age, luminosity, and mass, PSO J318.5-22 is an important benchmark for studies of young, directly imaged planets.

Based on observations obtained via Director's Discretionary Time at the Gemini Observatory, which is operated by the Association of Universities for Research in Astronomy, Inc., under a cooperative agreement with the NSF on behalf of the Gemini partnership: the National Science Foundation (United States), the National Research Council (Canada), CONICYT (Chile), the Australian Research Council (Australia), Ministério da Ciência, Tecnologia e Inovação (Brazil) and Ministerio de Ciencia, Tecnología e Innovación Productiva (Argentina). This work made use of Bucknell's Linux Computing Cluster maintained by the Engineering Computing Support Team. KNA and JFG acknowledge support from the Isaac J. Tressler Fund for Astronomy at Bucknell University.

\section{REFERENCES}

Allard, F., Hauschildt, P. H., Alexander, D. R., Tamanai, A., \& Schweitzer, A. 2001, ApJ, 556, 357

Allard, F., Homeier, D., Freytag, B., Sharp, C. M. 2012, EAS, 57,

Allers, K. N., \& Liu, M. C. 2013, ApJ, 772, 79

Asplund, M., Grevesse, N., Sauval, A. J., \& Scott, P. 2009, ARA\&A, 47, 481

Baraffe, I., Chabrier, G., Barman, T. S., Allard, F., \& Hauschildt, P. H. 2003, A\&A, 402, 701

Barman, T. S., Macintosh, B., Konopacky, Q. M., \& Marois, C. 2011, ApJ, 735, L39

Bell, C. P. M., Mamajek, E. E., \& Naylor, T. 2015, MNRAS, 454, 593

Berrut, J. P., \& Trefethen, L. N. 2004, SIAMR, 46, 501

Blake, C. H., Charbonneau, D., \& White, R. J. 2010, ApJ, 723, 684

Biller, B. A., Vos, J., Bonavita, M., et al. 2015, ApJ, 813, L23

Binks, A. S., \& Jeffries, R. D. 2014, MNRAS, 438, L11

Burrows, A., Hubbard, W. B., Lunine, J. I., \& Liebert, J. 2001, RvMP, 73, 719

Caffau, E., Ludwig, H.-G., Steffen, M., Freytag, B., \& Bonifacio, P. 2011, Sol. Phys., 268, 255

Chabrier, G., Baraffe, I., Allard, F., \& Hauschildt, P. 2000, ApJ, 542,464

Cushing, M. C., Vacca, W. D., \& Rayner, J. T. 2004, PASP, 116, 362

da Silva, L., Torres, C. A. O., de La Reza, R., et al. 2009, A\&A, 508,833

Elias, J. H., Joyce, R. R., Liang, M., et al. 2006, Proc. SPIE 6269,62694

Filippazzo, J. C., Rice, E. L., Faherty, J., et al. 2015, ApJ, 810, 158
Gagné, J., Lafrenière, D., Doyon, R., Malo, L., \& Artigau, É. 2014, ApJ, 783, 121

Geweke, J., 1992, Bayesian Statistics 4 (ed JM Bernardo, JO Berger, AP Dawid, and AFM Smith). Clarendon Press, Oxford, UK.

Hastings, W. K.,1970. Biometrika 57 (1): 97109.

Horne, K. 1986, PASP, 98, 609

King, J. R., Soderblom, D. R., Fischer, D., \& Jones, B. F. 2000, ApJ, 533, 944

Kraus, A. L., Shkolnik, E. L., Allers, K. N., \& Liu, M. C. 2014, AJ, 147, 146

Lagrange, A.-M., Gratadour, D., Chauvin, G., et al. 2009, A\&A, 493, L21

Livingston, W., \& Wallace, L. 1991, NSO Technical Report, Tucson: National Solar Observatory, National Optical Astronomy Observatory, 1991

Liu, M. C., Magnier, E. A., Deacon, N. R., et al. 2013, ApJ, 777, L20

Macintosh, B., Graham, J. R., Barman, T., et al. 2015, Science, 350,64

Males, J. R., Close, L. M., Morzinski, K. M., et al. 2014, ApJ, 786,32

Malo, L., Doyon, R., Feiden, G. A., et al. 2014, ApJ, 792, 37

Malo, L., Doyon, R., Lafrenière, D., et al. 2013, ApJ, 762, 88

Mamajek, E. E., \& Bell, C. P. M. 2014, MNRAS, 445, 2169

Mentuch, E., Brandeker, A., van Kerkwijk, M. H., Jayawardhana, R., \& Hauschildt, P. H. 2008, ApJ, 689, 1127

Metchev, S. A., Heinze, A., Apai, D., et al. 2015, ApJ, 799, 154

Metropolis, N., Rosenbluth, A.W., Rosenbluth, M.N., Teller, A.H., \& Teller, E., 1953, J. Chem. Phys., 21, 1087

Nelder, J. A., \& Mead, R., 1965, CompJ, 7, 308

Pecaut, M. J., Mamajek, E. E., \& Bubar, E. J. 2012, ApJ, 746, 154

Press, William H. and Teukolsky, Saul A. and Vetterling, William T. \& Flannery, Brian P. 2007, Numerical Recipes: The Art of Scientific Computing (3rd ed.; New York, NY: Cambridge University Press)

Radigan, J., Lafrenière, D., Jayawardhana, R., \& Artigau, E. 2014, ApJ, 793, 75

Saumon, D., \& Marley, M. S. 2008, ApJ, 689, 1327

Snellen, I. A. G., Brandl, B. R., de Kok, R. J., et al. 2014, Nature, 509, 63

ter Braak, C. \& Vrugt, J., 2008, Statistics and Computing, 18(4), 435-446.

Torres, C. A. O., Quast, G. R., Melo, C. H. F., \& Sterzik, M. F. 2008, Handbook of Star Forming Regions, Vol. 2, Young Nearby Loose Associations (The Southern Sky ASP Monograph Publications), Vol. 5, ed. B. Reipurth (San Francisco, CA: ASP), 757

Waring, E. 1779, RSPT, 69, 59

Zuckerman, B., Song, I., Bessell, M. S., \& Webb, R. A. 2001, ApJ, 562, L87

Zhou, Y., Apai, D., Schneider, G., Marley, M. S., \& Showman, A. P. 2015, ApJ, in press (arXiv:1512.02706) 